\title{
Triple and Double Photons Absorption Process and Down-Conversion Laser Emitting Investigation of Er-Ion Doped Microsphere
}

\author{
Yantang Huang $^{1 *}$, Peijing Zhang ${ }^{1}$, Yu Huang ${ }^{1}$, Changlei Guo ${ }^{1,2}$ \\ ${ }^{1}$ Physics and Information Engineering College, Fuzhou University, Fuzhou, China \\ ${ }^{2}$ Department of Electronic Engineering, Institute of Optoelectronic Technology, Xiamen University, Xiamen, China \\ Email: "huangyantang@fzu.edu.cn
}

Received October 8, 2013; revised November 5, 2013; accepted November 27, 2013

Copyright (C) 2013 Yantang Huang et al. This is an open access article distributed under the Creative Commons Attribution License, which permits unrestricted use, distribution, and reproduction in any medium, provided the original work is properly cited.

\begin{abstract}
We report a readily and cheap method to build taper optical fiber-Er ${ }^{3+}$ doped microsphere platform to investigate upconversion fluorescence emission and down-conversion laser oscillation with low threshold pump power. We demonstrate to dope $\mathrm{Er}^{3+}$ into silica microsphere surface by dipping a single-taper optical fiber into a certain concentration of erbium nitrate solution $\left(\mathrm{Er}\left(\mathrm{NO}_{3}\right)\right)$, then dry it and use the electrical-arc of the optical fiber splicer to melt the tip of taper fiber to form the $\mathrm{Er}^{3+}$-doped silica microsphere due to surface tension induced. We also present a $\mathrm{HF}$ acid etching setup to fabricate low loss biconical optical taper fibers. We demonstrate the $\mathrm{Er}^{3+}$ doped silica microsphere triple photons and dual photons absorption process of up-conversion fluorescence emission and down-conversion laser oscillation spectra by using the optical tapered fiber to couple $976 \mathrm{~nm} / 1534 \mathrm{~nm}$ pump light source.
\end{abstract}

Keywords: Triple Photons Absorption Process; $\mathrm{Er}^{3+}$ Doped Microsphere; Taper Optical Fiber; Micro-Laser

\section{Introduction}

Rare earth doped materials have been widely used for photonic applications including lasers [1], optical fiber amplifiers [2], and laser display [3], optical anti-counterfeiting [4], and anti-Stokes fluorescence laser cooling [5]. Erbium with abundant energy level structures was particularly studied for erbium-doped fiber amplifier (EDFA) [6], optical fiber lasers [1] and up-conversion emissions [7]. In surface-tension induced silica microspheres light can propagate around the inner surface with very low loss due to atomic-scale smooth surface in the manner of total internal reflection, called "whispering-gallery" modes (WGMs). The WGMs attained not only the highest optical quality-factors $(\mathrm{Q})$ (i.e. the temporal confinement of light) [8-10], but also the light is spatially confined, exhibiting small mode volume and high energy density of the optical mode. The small mode volume and long photon storage time can be used for a variety of studies ranging from fundamental physics such as cavity Quantum Electrodynamics [11] to more applied areas nonlinear optical studies $[9,10]$, high-sensitivity transducers for

${ }^{*}$ Corresponding author. biochemical sensing [12], low threshold light emission and narrow line-width lasers [8], as strong resonant buildup of energy in micro-scale volumes significantly reduces the threshold for light emission to occur. Silica is good host for some rare earth ions including Er (erbium), $\mathrm{Yb}$ (ytterbium), Tm (thulium), Ho (holmium) [7,13,14] etc. When silica microspheres are doped with certain number of rare-earth ions, light can be emitted by coupling pump light source with a tapered fiber $[15,16]$. Microsphere and biconical tapered fiber coupled system is good candidate for study of rare earth doped material light emission. A biconical tapered fiber not only can import pump light into a microsphere, but also can export emitted light into a single mode fiber, which is very convenient for readily measurement.

In this paper, microspheres are fabricated by the electrical-arc melting of the optcal fiber splicer. The diameter of the microsphere ranges from the tens of to the hundreds of micron. A fiber taper made of SMF-28 fiber by HF etching method was used to couple the pump light with emission light or laser out of the microsphere. A semiconductor laser diode at $976 \mathrm{~nm}$ and TLS laser $(1470-1580 \mathrm{~nm})$ are employed to directly excite the Er 
(erbium)-ion doped silica microsphere.

\section{Er-Ion Doped Microsphere and Optical Taper Fiber Fabrication}

At first, we stripped off the coating of a single mode fiber for $3 \mathrm{~cm}$ long. The bare optical fiber was carefully cleaned by acetone and alcohol, then heated with the flame of alcohol lamp and pulled with a steady speed from the ends until it was fractured in the middle, leaving two single tapered fibers. The diameter and length of a taper are related to the pulling speed. In our experiments, the tip diameters of tapers were less than 20 micron. In order to dope Er-ions into silica, a tapered fiber was dipped into a certain erbium nitrate solution, its concentration was about $10^{-5}-10^{-4} \mathrm{~mol} / \mathrm{ml}$. Then it was fixed between two thermostability electrodes of splicer (Figure 1B(a)). After the electrodes discharged under the control of circuit, the taper tip was melted in several seconds. After several times of electrical-arc melting, an Er-ion doped silica microsphere was fabricated (Figure 1B(c)), which exhibits a near atomic scale surface finish due to surface-tension-induced.

We present a new HF acid etch setup to fabricate low loss biconical optical tapered fibers with waist diameter between $1.0 \mu \mathrm{m}$ to $2.5 \mu \mathrm{m}$. Instead of the acid baths and an uncontrollable micro-droplet of HF [18], we design a etching groove to obtain different etching rate along the optical fiber as shown in Figure 2.

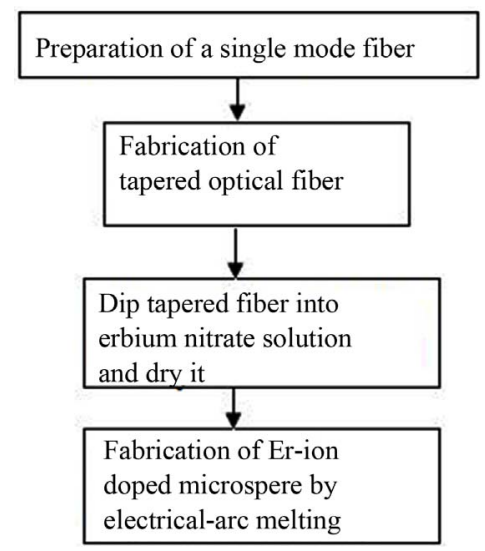

(A)

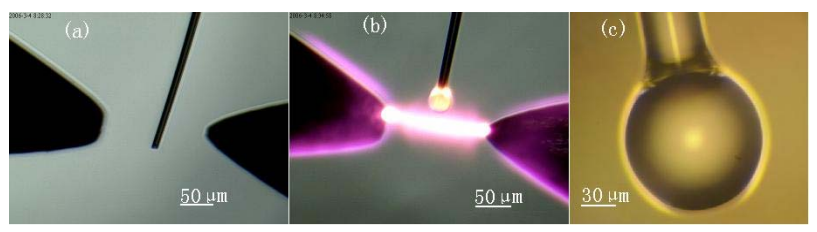

(B)

Figure 1. Fabrication of an Er-ion doped silica microsphere. (A) The flow chart of the Er-ion doped microsphere; (B) Electrical-arc melting to fabricate Er-doped microsphere [17].
The etch groove was made from plastic material. There were an oval groove in the mid and two small Vshape grooves in the two sides respectively. As the oval groove was injected with $0.05 \mathrm{ml}-0.06 \mathrm{ml}$ buffered HF (BHF) acid and the two small V-shape grooves were injected full of deioned water, the HF acid solution diffused toward the V-grooves, its concentration decreased gradually along the grooves to etch the fiber to form a graded diameter profile. The V-groove must be small enough to control the diffusion. We terminated an etch by extracting the HF using a pipette and flushing the fiber with deionized water and acetone.

The surface morphology of fabricated tapered fiber was detected by $400 \times$ optical microscope and atomic force microscope (AFM) as shown in Figure 3. The roughness of the surface was less than $1 \mathrm{~nm}$. The tapered fiber exhibited excellent surface smoothness.

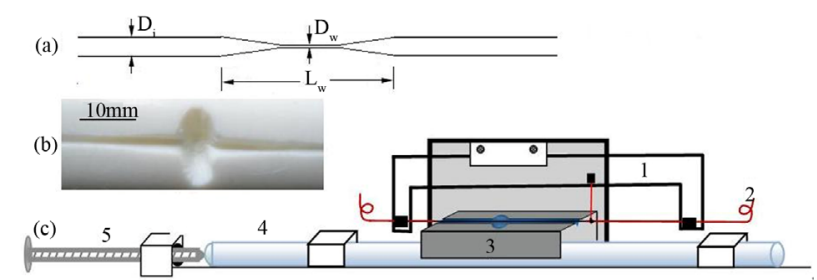

Figure 2. The setup of using HF acid to fabricate low loss biconical optical tapered. (a) Schematic of a biconical tapered fiber. $L_{w}$ is the taper length (contracting and expanding regions). $D_{i}$ is the original fiber diameter $(125 \mu \mathrm{m})$ of a SMF and $D_{w}$ is the diameter of the waist; (b) The etching groove with an oval in the mid and two small $V$-shape groove on both sides; (c) The schematic of the etching apparatus: 1. U-shape frame for fixing fiber; 2 . Optical fiber; 3. Etching groove; 4. Moveable rod for detaching the etching groove and the final taper fiber; 5 . Screw bolt.
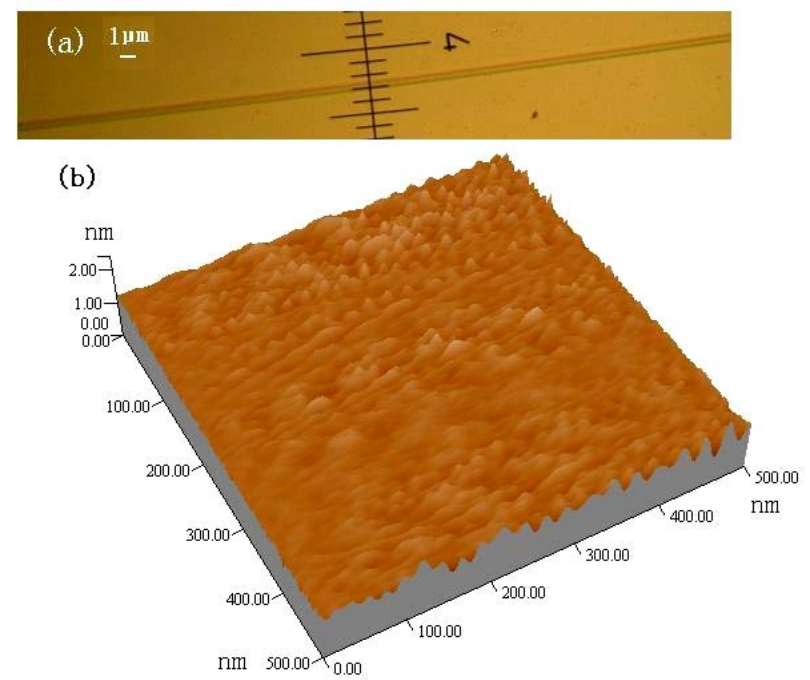

Figure 3. (a) Final fabricated taper: an optical micrograph of the waist under $400 \times$ optical microscope; (b) The surface morphology of the tapered fiber under AFM. 


\section{Er-Ion Doped Microsphere Light Emission Measurement and Analysis}

\subsection{Up-Conversion Fluorescence Emission}

Figure 4 is the schematic diagram of measurement setup. An Er-ion doped silica microsphere was firstly pumped by a tunable laser source at $1534 \mathrm{~nm}$. Pump light was imported through single mode fiber to biconical tapered fiber, the tapered fiber it tangent coupled with a microsphere, and the emission light (green fluorescence) and the residual $976 \mathrm{~nm}$ light was also exported through the single mode fiber.

Figure 5(A) shows the variation of the upconversion fluorescence spectrum for a $73 \mu \mathrm{m}$ Er-doped microsphere as the pump power is varied from 0.52 to $4.02 \mathrm{~mW}$ (the concentration of erbium nitrate solution is $1.7 \times 10^{-4}$ $\mathrm{mol} / \mathrm{ml}$, the pumped wavelength is $1534 \mathrm{~nm}$ ) at room temperature.

The peak wavelength is related to ${ }^{4} \mathrm{~S}_{3 / 2} \rightarrow{ }^{4} \mathrm{I}_{15 / 2}$ transition of $\mathrm{Er}^{3+}$ ion (Figure 6(b)), which is a triple-photon absorption process [19]. In fact, the microsphere can be excited by pump light from 1470 to $1580 \mathrm{~nm}$ with suitable pump power. As the microsphere was pumped with the wavelength of $976 \mathrm{~nm}$ using a DFB $980 \mathrm{~nm}$ laser source. We measured the dual photons absorption process of up-conversion fluorescence emission. Figure 5(B) shows its luminescence spectra from 400 to $660 \mathrm{~nm}$ with different pump power, the wavelength of $660 \mathrm{~nm}, 546$ $\mathrm{nm}$ and $410 \mathrm{~nm}$ emissions were achieved from the transitions, ${ }^{4} \mathrm{~F}_{9 / 2} \rightarrow{ }^{4} \mathrm{I}_{15 / 2},{ }^{4} \mathrm{~S}_{3 / 2} \rightarrow{ }^{4} \mathrm{I}_{15 / 2}$, and ${ }^{2} \mathrm{H}_{9 / 2} \rightarrow{ }^{4} \mathrm{I}_{15 / 2}$, respectively [17]. Figure 6(a) was up-conversion luminescence intensity as a function of the pump power at 976 $\mathrm{nm}$.

The slopes of best fitting straight lines for $410 \mathrm{~nm}, 548$

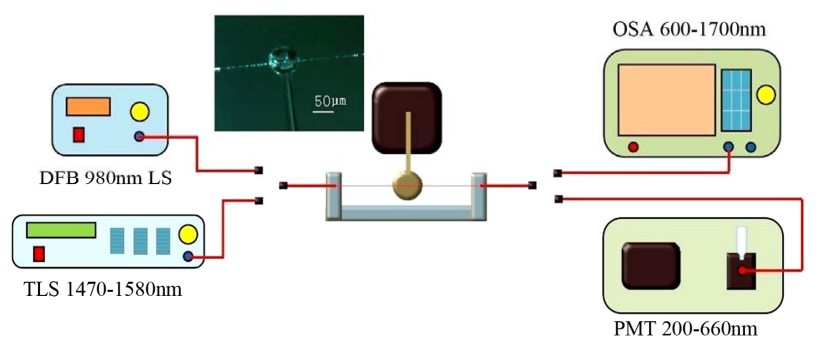

Figure 4. Schematic diagram of measurement setup. DFB $980 \mathrm{~nm}$ LS has a central pump wavelength at $976 \mathrm{~nm}$. TLS is a tunable laser source with tunable pump wavelength from 1470 to $1580 \mathrm{~nm}$. An optical spectrum analyzer (OSA) (range from 600 to $1700 \mathrm{~nm}$ ) with resolution of $0.2 \mathrm{~nm}$ was used to measure the laser emission. PMT represents a grating spectrometer attached to a photomultiplier tube with detection range from 200 to $660 \mathrm{~nm}$. In the mid, the microsphere is fixed on a three axies manual control stage to touch the biconical tapered fiber fixed on a fiber holder (U-shaped). The inset is a photomicrograph of the side view of an erbium doped microsphere coupled by a fiber taper.

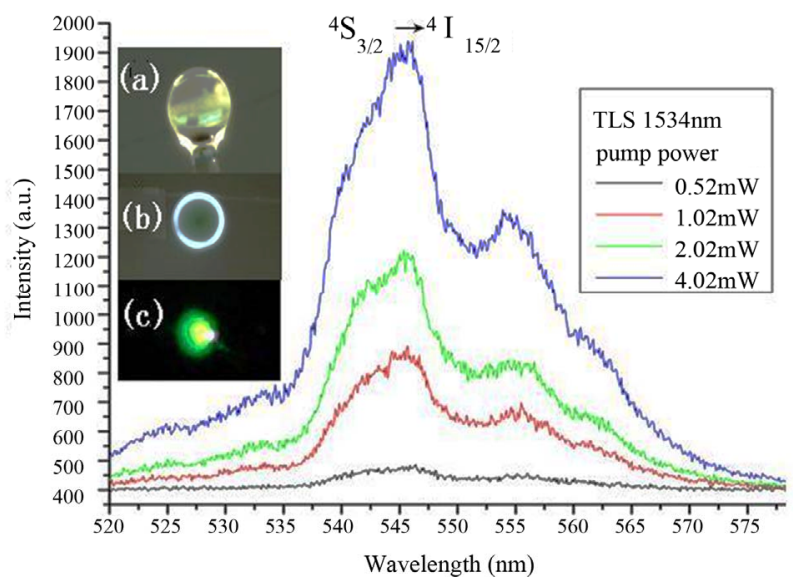

(A)

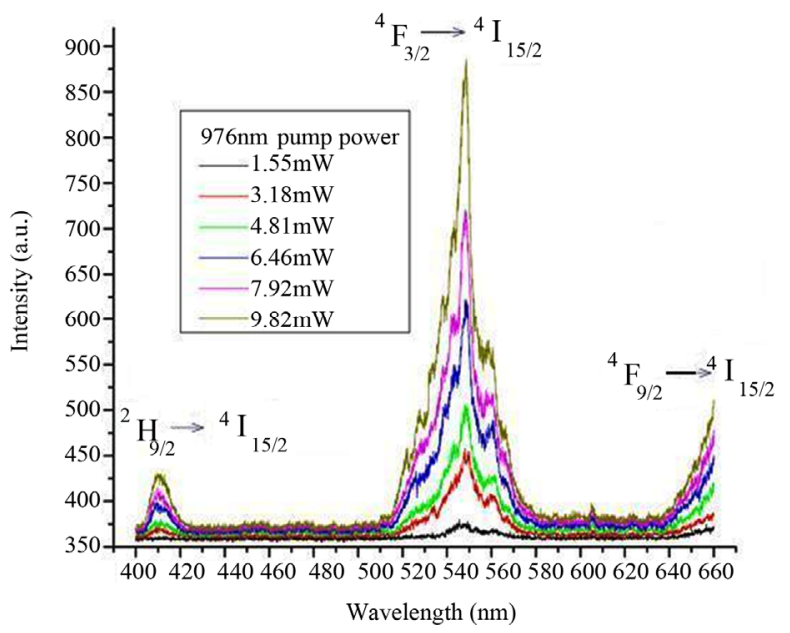

(B)

Figigure 5. (A) Room temperature luminescence spectra of the ${ }^{4} S_{3 / 2} \rightarrow{ }^{4} I_{15 / 2}$ transition of $\mathbf{E r}^{3+}$ ion around the center wavelength of $540 \mathrm{~nm}$ with the pump power from $0.52 \mathrm{~mW}$ to $4.02 \mathrm{~mW}$ at pump wavelength of $1534 \mathrm{~nm}$. Inset; (a) and (b) are top and vertical view of a microsphere with green luminescence, respectively. Inset (b) the out layer ring of light is $976 \mathrm{~nm}$ light; (c) is picture taken by a digital camera from the tail of an optical fiber; (B) Room temperature luminescence spectra of $\mathrm{Er}^{3+}$ from 400 to $660 \mathrm{~nm}, 976 \mathrm{~nm}$ pump power was from 1.55 to $9.82 \mathrm{~mW}$.

$\mathrm{nm}$ and $660 \mathrm{~nm}$ luminescence intensity are 1.91, 1.96 and 1.44 , respectively, which are two-photon processes. The $410 \mathrm{~nm}$ light emission is also dual-photon process of 745 $\mathrm{nm}$ noise light pump, which was existing in the DFB 980 nm laser source, which was $27 \mathrm{~dB}$ weaker than $976 \mathrm{~nm}$ pump light.

\subsection{Down-Conversion Laser Oscillation}

When the microsphere was pumped with the wavelength of $976 \mathrm{~nm}$, we have also measured the down conversion laser oscillation; a typical laser spectra is presented in Figure 7. The microsphere diameter was $139 \mu \mathrm{m}$, the concentration of erbium nitrate solution was $2.87 \times 10^{-5}$ 


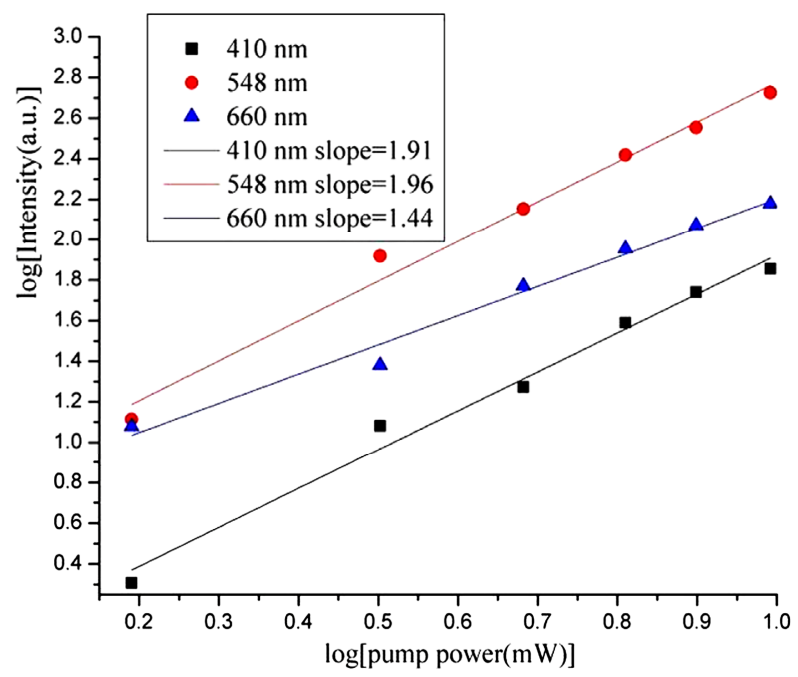

(a)

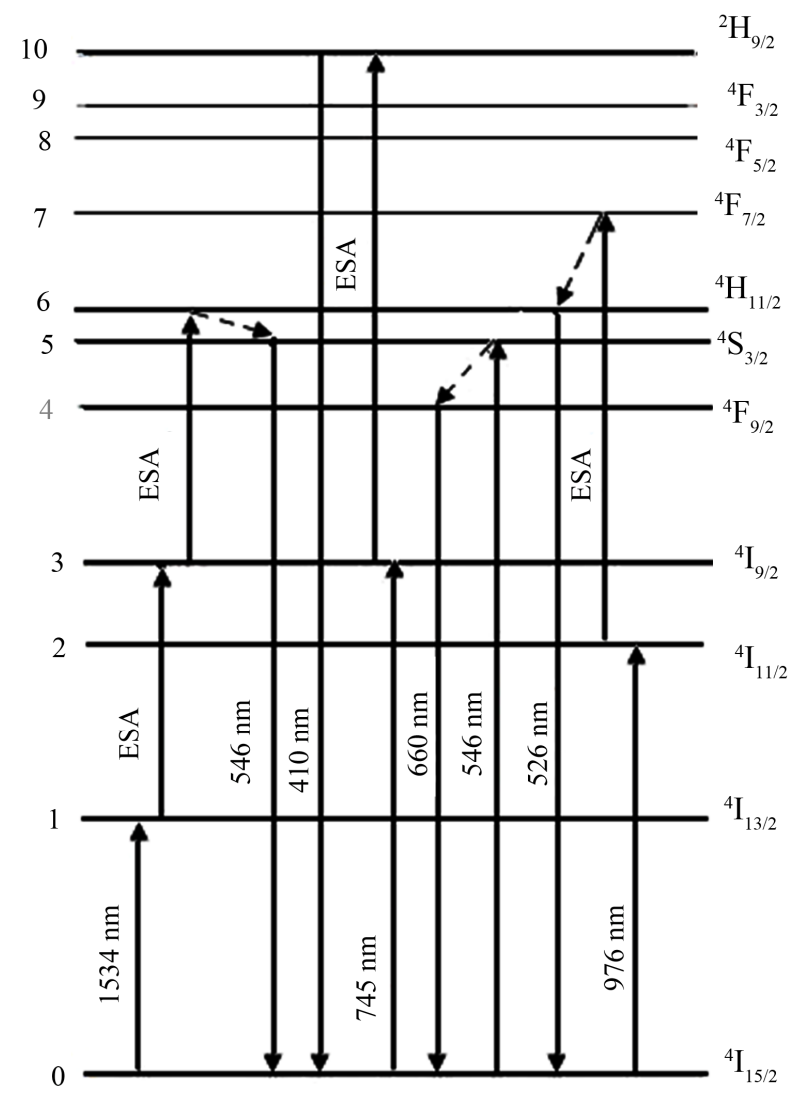

(b)

Figure 6. (a) Up-conversion luminescence intensity as a function of the pump power at $976 \mathrm{~nm}$. The slopes of best fitting straight lines for $410 \mathrm{~nm}, 548 \mathrm{~nm}$ and $660 \mathrm{~nm}$ luminescence intensity are 1.91, 1.96 and 1.44 , respectively; (b) $\mathrm{Er}^{3+}$ energy level diagram of up conversion luminescence [17]. ESA (excited state absorption).

$\mathrm{mol} / \mathrm{ml}$. Single frequence emission (within the resolution of the OSA) was most often observed, however, as increased pumping levels it was always possible to in-

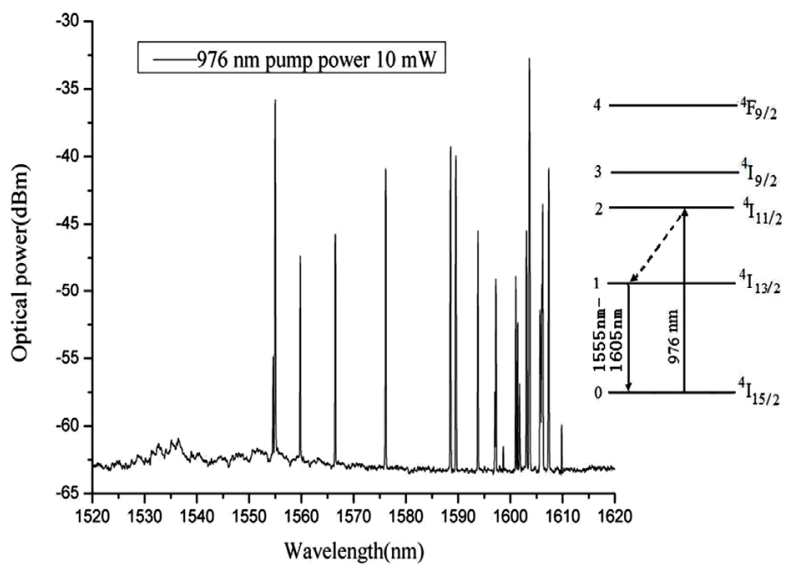

Figure 7. Emission spectrum of a $\mathbf{E r}^{3+}$ doped microsphere laser with diameter $139 \mu \mathrm{m}, 976 \mathrm{~nm}$ pump power is $10 \mathrm{~mW}$, and inset illustrates the energy diagram of the electronic state of $\mathbf{E r}^{3+}$.

duce oscillation in other longitudinal modes. The wavelength of $1550 \mathrm{~nm}-1610 \mathrm{~nm}$ down conversion laser oscillation were achieved from the electronic state transitions ${ }^{4} \mathrm{I}_{13 / 2} \rightarrow{ }^{4} \mathrm{I}_{15 / 2}$.

\section{Conclusion}

We use readily and cheap method to fabricate Er-ion doped silica microspheres with diameters from 70 to 150 micron, and biconical tapered fibers with waist diameter less than 2 micron and insertion loss less than $0.5 \mathrm{~dB}$. Using microsphere and tapered fiber coupled system, we observed triple photons absorption process of $520 \mathrm{~nm}$ $580 \mathrm{~nm}$ green up-conversion light by $1534 \mathrm{~nm}$ pumping, and dual photons absorption process of violet, green, red up-conversion light by $976 \mathrm{~nm}$ pumping. At the same time, pumped with $976 \mathrm{~nm}$ DFB laser diode, 1550 - 1610 nm laser oscillation was observed. The pump power was only several microwatts, and up conversion luminescence was easily observed and measured. Using this coupled system, we can study the luminescence emission of the rare earth ions doped material. The readily fabricating manners of rare earth doped microsphere and biconical tapered fiber are good candidate platform for the study of rare earth doped material light emission.

\section{REFERENCES}

[1] X. Peng, F. Song, S. Jiang and N. Peyghambarian, M. Kuwata-Gonokami and L. Xu, Applied Physics Letters, Vol. 82, 2003, p. 1497. http://dx.doi.org/10.1063/1.1559653

[2] T. Kasamatsu, Y. Yano and T. Ono, IEEE of Photonics Technology Letters, Vol. 13, 2001, pp. 31-33. http://dx.doi.org/10.1109/68.903211

[3] M. Y. Liu and R. P. Zhang, Chinese Journal of Laser, Vol. 38, 2011, p. 10. 
[4] H. H. Jia, X. A. Zhang, H. L. Zhang, F. Wang and S. L. Chang, Chinese Journal of Lasers, Vol. 36, 2009, pp. 393-395.

[5] J. Fernandez, A. Mendioroz, A. J. Garcia, R. Balda and J. L. Adam, Physical Review B, Vol. 62, 2000, pp. 32133217. http://dx.doi.org/10.1103/PhysRevB.62.3213

[6] L. L. Yi, L. Zhan, W. S. Hu, Q. X. Tang and Y. X. Xia, Optics Express, Vol. 14, 2006, pp. 570-574. http://dx.doi.org/10.1364/OPEX.14.000570

[7] N.-K. Chen, P.-W. Kuan, J. J. Zhang, et al., Optics Express, Vol. 18, 2010, pp. 25615-25626. http://dx.doi.org/10.1364/OE.18.025615

[8] L. Yang, D. K. Armani and K. J. Vahalab, Applied Physics Letters, Vol. 83, 2003, pp. 825-826. http://dx.doi.org/10.1063/1.1598623

[9] S. M. Spillane, T. J. Kippenberg and K. J. Vahala, Nature, Vol. 415, 2002, pp. 621-623. http://dx.doi.org/10.1038/415621a

[10] B. Min, T. J. Kippenberg and K. J. Vahala, Optics Letters, Vol. 28, 2003, pp. 1507-1509. http://dx.doi.org/10.1364/OL.28.001507

[11] H. Rokhsari and K. Vahala, Physical Review Letters, Vol. 92, 2004, p. 253. http://dx.doi.org/10.1103/PhysRevLett.92.253905
[12] Z. P. Cai and H. Y.Xu, Sensors and Actuators A: Physical, Vol. 108, 2003, pp. 187-192. http://dx.doi.org/10.1016/j.sna.2003.07.008

[13] S. D. Jackson, IEEE Photonics Technology Letters, Vol. 18, 2006, pp. 1885-1887. http://dx.doi.org/10.1109/LPT.2006.881686

[14] J. J. Koponen, M. J. Söderlund, H. J. Hoffman and S. K. T. Tammela, Optics Express, Vol. 14, 2006, pp. 1153911544. http://dx.doi.org/10.1364/OE.14.011539

[15] M. Cai, O. Painter and K. J. Vahala, Optics Letters, Vol. 25, 2000, pp. 1430-1432. http://dx.doi.org/10.1364/OL.25.001430

[16] C.-H. Dong, Y.-F. Xiao, Z.-F. Han, G.-C. Guo, X. S. Jiang, L. M. Tong, C. Gu and H. Ming, IEEE Photonics Technology Letters, Vol. 20, pp. 342-344.

[17] P.-J. Zhang, Y. Huang, C.-L. Guo and Y.-T. Huang, Acta Physica Sinica, Vol. 62, 2013, Article ID: 224207.

[18] E. J. Zhang, W. D. Sacher and J. K. S. Poon, Optic Express, Vol. 18, 2010, pp. 22593-22598. http://dx.doi.org/10.1364/OE.18.022593

[19] L.-P. Lu, X.-Y. Zhang, K. Gong, C. Zu and Y. Bi, Chinese Journal of Luminescence, Vol. 32, 2011, pp. 237240. 